\title{
THE MAXIMUM MOMENT OF INERTIA OF NEUTRON STARS AND ITS IMPLICATIONS FOR PULSAR OBSERVATIONS
}

\author{
Pawez Haensel \\ Copernicus Astronomical Center
}

\begin{abstract}
A simple approximate formula, expressing the maximum moment of inertia of a neutron star as a function of the mass and radius of the configuration with a maximum allowable mass, is shown to be a quite precise representation of the results obtained for a broad set of equations of state of dense matter. The resulting possible observational constraints in the mass-radius plane for neutron star models are discussed.
\end{abstract}

The moment of inertia of a neutron star plays an important role in phenomenological models of pulsars. The total energy expenditure per unit time, $\dot{E}_{\text {tot }}$, including the observed luminosity and the power needed to accelerate the material of the surrounding nebula, is accounted for by the slowing down of the neutron-star rotation. In the standard case of the rigid rotation, $\dot{E}_{\text {tot }}$ can be related to the measured rotation frequency, $\Omega$, and its time derivative, $\dot{\Omega}$, by $\dot{E}_{\mathrm{tot}}=-\dot{E}_{\mathrm{rot}}=-I \Omega \dot{\Omega}$, where $I$ is the moment of inertia for rigid rotation. This leads to an observational estimate $I_{\text {obs }}=-\dot{E}_{\text {tot }} / \Omega \dot{\Omega}$, provided we have a reliable estimate of $\dot{E}_{\text {tot }}$.

Even in the case of slow ${ }^{1}$ rigid rotation, the calculation of the moment of inertia of a neutron star is complicated by general relativistic effects, such as dragging of the local inertial frames. An important feature stemming from general relativity is the existence, for a fixed equation of state (EOS) of superdense matter, of the maximum moment of inertia, $I_{\max }$. This maximum value is reached for a neutron-star mass below the maximum allowable mass, $M_{\max }$. The parameters of the neutron-star configuration corresponding to $I_{\max }$ (or $M_{\max }$ ) are determined by the properties of superdense matter well above the normal nuclear density, $\rho_{0}=2.5 \times 10^{14} \mathrm{~g} \mathrm{~cm}^{-3}$. Unfortunately, the actual properties of matter for, say, $\rho>2 \rho_{0}$, are unknown, and thus it is of the utmost importance to look for constraints on the parameters of neutron stars, which have a universal character and are independent of the particular form of the EOS of dense matter.

Generally, the scatter of the values of $I_{\max }$, obtained for various EOS of dense matter, is much larger than that for the values of $M_{\max }$. This feature of neutron-star models was pointed out seventeen years ago by Carter and Quintana (1973), who confronted the then existing EOS of dense matter

${ }^{1} \Omega^{2} \ll(c / R)^{2} G M / R c^{2}, R \Omega \ll c$, conditions which are satisfied for pulsar period greater than $1 \mathrm{~ms}$ (which are now considered to be very unrealistic from the point of view of the theory of dense matter) with the scarce data then available about the energetics of the Crab pulsar. In this way they were able to eliminate the softest EOS of their set. Upper bounds on the neutron-star moment of inertia, based only on general relativity and causality, have been derived by Hartle (1978). These theoretical upper bounds are, however, too large to be of practical significance.

In the present note we discuss an approximate but surprisingly precise relation between $I_{\max }$ and the mass and radius of the configuration with the maximum allowable mass, $M_{\max }$, and $R_{\max }$ : $I_{\max } \simeq \alpha M_{\max } R_{\max }^{2}$, where $\alpha$ does not depend on the EOS, and can be calculated by fitting this relation to the existing numerical results for various EOS of dense matter. Our fit has been carried out for a set of nineteen EOS, which included a broad set of EOS studied by Arnett and Bowers (1977), EOS with first order phase transitions above $\rho_{0}$ (Haensel and Prószyński 1982), EOS with a large percentage of hyperons (Glendenning 1985), and strange stars built of self-bound quark matter (Haensel, Zdunik, and Schaeffer 1986). A least squares fit by minimization of the sum of the squares of the relative deviations yields, with $\chi^{2}=0.032$, the formula

$$
I_{\max , 45}=0.984 \frac{M_{\max }}{\mathrm{M}_{\odot}}\left(\frac{R_{\max }}{10 \mathrm{~km}}\right)^{2},
$$

where $I_{45}$ is the moment of inertia in units of $10^{45} \mathrm{~g} \mathrm{~cm}^{2}$. This formula reproduces the values of $I_{\max }$ typically within a few percent (figure 1 ). The quality of the fit is surprisingly good, especially in view of the fact that the values of $I_{\max , 45}$ change by an order of magnitude when passing from very soft $\operatorname{EOS}\left(I_{\max , 45} \sim 0.5\right)$ to very stiff ones $\left(I_{\max , 45 \sim 5)}\right.$. It should be stressed that $I_{\max }$ in eq. (1) refers to a different neutron star configuration than that with $M_{\max }$ and $R_{\max }$. Had we tried to fit $I_{\max }$ with 


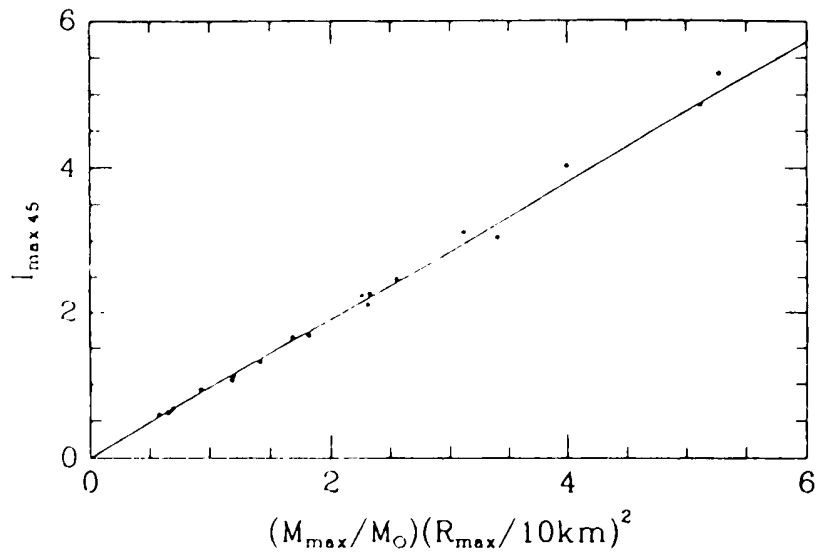

Figure 1 The least squares fit to the dependence of $I_{\max }$ on $M_{\max } R_{\max }^{2}$. Each point corresponds to one of the EOS, listed in the text.

the actual values of $M$ and $R$ of the configuration with the maximum value of $I$, the fit would be rather poor, with the numerical coefficient equal to $0.83, \chi^{2}=0.26$, and the relative deviations typically $\sim 10 \%$ and sometimes as large as $20 \%$. In view of this we think that eq.(1) has some feature of universality.

Eq.(1) allows us to formulate a practical constraint on the $M-R$ relation for neutron stars, resulting from the detection of a pulsar with moment of inertia $I_{\text {obs. }}$. To be consistent with this observational fact, an EOS should satisfy

$$
\frac{M_{\max }}{M_{\odot}}>1.02 I_{\text {obs, } 45}\left(\frac{R_{\max }}{10 \mathrm{~km}}\right)^{-2}
$$

Until now, the only observational estimates of $I_{\text {obs }}$ are those for the Crab pulsar $I_{\text {Crab }}$. The total power emitted by the Crab Nebula in the form of optical, ultraviolet and $\mathrm{X}$-ray radiation has been recently estimated as

$$
\dot{E}_{\mathrm{rad}}=2 \times 10^{38}(d / 2.0 \mathrm{kpc})^{2} \mathrm{erg} \mathrm{s}^{-1},
$$

where $d$ is the distance of the nebula (Lozinskaya 1986). This luminosity should be accounted for by the loss of rotational energy of the Crab pulsar, $\dot{E}_{\text {rot }}=I_{\text {Crab }} \Omega \dot{\Omega}$. Using $\Omega \Omega=-4.59 \times 10^{-7} \mathrm{~s}^{-3}$, we thus obtain $I_{\mathrm{Crab}, 45}>0.4(d / 2.0 \mathrm{kpc})^{2}$, so that assuming the widely accepted value of the distance, $d=2.0 \mathrm{kpc}$, we get a lower bound $I_{\text {Crab }, 45}^{(1)}=0.4$. More detailed analysis of the energetics of the Crab Nebula (see, e.g., Manchester and Taylor 1977) allows one, in principle, to set the lower limit on $I_{\max }$ much higher. The usually quoted value of the total power required to support the accelerated expansion of the Crab Nebula $\dot{E}_{\exp }$ is $4 \times 10^{38} \mathrm{erg} \mathrm{s}^{-1}$ (Manchester and Taylor 1977). However, such an estimate of $\dot{E}_{\text {exp }}$, obtained using the very crude approximation of spherical symmetry, is based on many assumptions. The most important of these

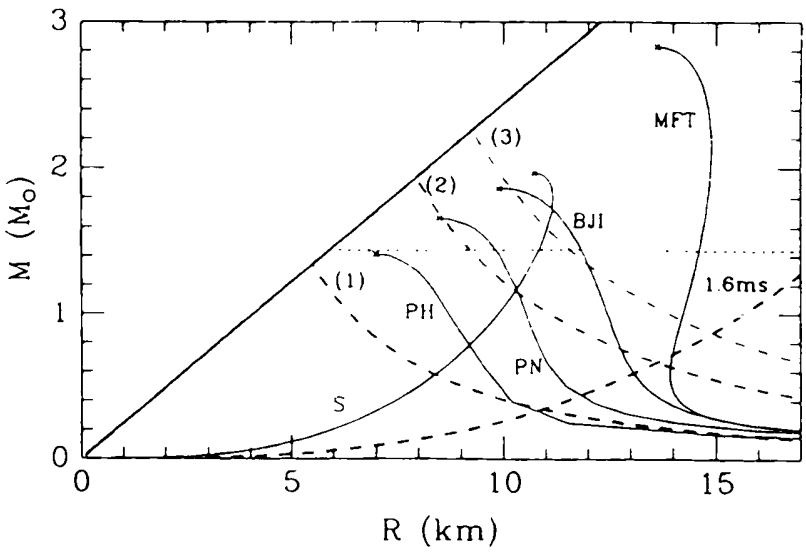

Figure 2 Allowed regions in the $M-R$ plane for nonrotating neutron star models. Examples of configurations with the maximum allowable mass are denoted by crosses. Configurations lying to the left of the heavy solid line are prohibited by general relativity and causality. The $M(R)$ curves for the various EOS are: MFTmean field theory, model 0.17 of Haensel et al. (1981); BJI-model I of Bethe and Johnson (Malone, Johnson, and Bethe 1975); PN-Pandharipande EOS for neutron matter (Pandharipande 1971a); PH-Pandharipande EOS for hyperonic matter (Pandharipande 1971b); S-model SQ of strange quark stars (Haensel, Zdunik, and Schaeffer 1986). The dotted horizontal segment corresponds to the mass of PSR 1913+16. For the further explanation, see the text.

assumptions are: $d=2.0 \mathrm{kpc}$, the value of the acceleration of the expansion taken as the average over the whole expansion period $\left(10^{-3} \mathrm{~cm} / \mathrm{s}^{2}\right)$, the mass of the nebula taken to be $1 \mathrm{M}_{\odot}$, the present radius of the expanding shell taken as $1 \mathrm{pc}$, the velocity of expansion $1500 \mathrm{~km} / \mathrm{s}$, and the density of the interstellar medium swept by the expanding envelope assumed to be $n_{\mathrm{H}}=0.2 \mathrm{~cm}^{-3}$. With these parameters, assuming $\dot{E}_{\mathrm{rot}}=-\dot{E}_{\mathrm{rad}}-\dot{E}_{\text {exp }}$, we get $I_{\text {Crab, } 45}^{(2)}=1.2$. As can be seen in figure 1 , this estimate of $I_{\text {Crab }}$ excludes a significant number of very soft EOS of dense matter. The actual uncertainty in the value of $I_{\text {Crab }}$ obtained from $\dot{E}_{\text {rot }}=-\dot{E}_{\text {rad }}-\dot{E}_{\text {exp }}$, however, is expected to be very large. For example, the uncertainty in $d$ is rather large (see, e.g., Davidson and Fesen 1985). Also, the total mass of the nebula is very poorly known.

In getting the estimate $I_{\text {Crab }}^{(2)}$, the value of $1 \mathrm{M}_{\odot}$ for the mass of the Crab nebula (not including the pulsar) has been assumed; this was a "standard" estimate of the nebula mass by the end of sixties (see, e.g., Minkowski 1968). More recent analysis based on absolute emission-line fluxes from the nebula suggests that the mass of the nebula, out to a radius of $1.6 \mathrm{pc}$ (and not counting the pulsar), is more likely $2-3 \mathrm{M}_{\odot}$ (Davidson and Fesen 1985). Using the same values of the remaining parameters as those used to get $I_{\text {Crab }}^{(2)}$, we obtain $I_{\text {Crab, 45 }}=1.9-2.5$. This would suggest $I_{\text {Crab, 45 }}>I_{\text {Crab }}^{(3)}=1.9$, eliminating 
a significant fraction of EOS represented by points in figure 1 . Constraints resulting from several estimates of $I_{\text {Crab }}$ are shown in figure 2. The allowed region in the $M-R$ plane is bourided from the left by the line corresponding to the maximum redshift of non-rotating neutron-star models, based on a causal EOS [velocity of sound $=(d P / d \rho)^{1 / 2} \leq c$ ] (Lindblom 1984). This absolute limit corresponds to $2 G M / R c^{2} \leq 0.72$. A neutron star should be sufficiently compact to rotate stably at the period of the most rapid millisecond pulsar, PSR 1937+214 (Shapiro, Teukolsky, and Wasserman 1983). This excludes neutron-star configurations lying below the heavy dashed line marked " $1.6 \mathrm{~ms}$ ", leading to a rather weak constraint on neutron-star models. If a pulsar with $I_{\text {obs }}$ has been observed, then the configurations with maximum allowable mass should lie above the line $M=1.02 I_{\text {obs, } 45}(R / 10 \mathrm{~km})^{-2}$. The constraints resulting from several estimates of $I_{\text {Crab }}$ are represented by dashed segments of hyperbolas, the confidence in the constraint increasing with the line thickness. The lower bound on $I_{\text {Crab }}, I_{\text {Crab }}^{(1)}$ [heavy dashed line (2) in figure 2], does not yield a significant constraint on the neutron-star models. On the coritrary, the estimate $I_{\text {Crab }}^{(2)}$ [medium heavy dashed line (2) in figure 2] yields quite a strong constraint on the softness of the EOS of dense matter. Specifically, for $R_{\max }<9.2 \mathrm{~km}$ the value of $I_{\text {Crab }}^{(2)}$ puts a stronger constraint on the minimum stiffness of the EOS than that resulting from the measured mass of PSR $1913+16\left(M_{\max }>1.44 \mathrm{M}_{\odot}\right.$, Taylor and Weisberg 1989). A still larger estimate, $I_{\text {Crab }}^{(3)}$ (thin dashed line (3) in figure 2), puts a rather strong constraint on the EOS, allowing only stiff ones. It should be stressed, however, that the actual uncertainty in our estimates of $I_{\text {Crab }}$ is expected to be very large. Despite the very scarce present information about the moments of inertia of neutron stars, we hope that the constraint derived in the present note can be potentially useful in constraining the EOS of dense matter. When passing from the softest EOS to the stiffest one, $M_{\max }$ increases by a factor of two, whereas $I_{\max }$ increases by a factor of ten. Clearly, $I_{\max }$ is much more sensitive to the EOS than $M_{\max }$. This is why future determinations of the moments of inertia of pulsars can lead to rather strong constraints on the theories of superdense matter.

Acknowledgment: The computations on which this work is based have been made on an IBM/XT compatible microcomputer on loan from Princeton University. 